\title{
DISCOVERY AND ANALYSIS OF ACTIVITY PATTERN CO- OCCURRENCES IN BUSINESS PROCESS MODELS
}

\author{
Jean Michel Lau, Cirano Iochpe \\ Informatics Institute, Federal University of Rio Grande do Sul, 9500 Bento Gonçalves Av., Porto Alegre, Brazil \\ jmlau@inf.ufrgs.br, ciochpe@inf.ufrgs.br \\ Lucinéia Heloisa Thom, Manfred Reichert \\ Institute of Databases and Information Systems, University of Ulm, Germany \\ lucineia.thom@uni.ulm.de,manfred.reichert@uni-ulm.de
}

Keywords: Business process modeling, workflow activity patterns, knowledge discovery, data mining, reuse.

\begin{abstract}
Research on workflow activity patterns recently emerged in order to increase the reuse of recurring business functions (e.g., notification, approval, and decision). One important aspect is to identify pattern cooccurrences and to utilize respective information for creating modeling recommendations regarding the most suited activity patterns to be combined with an already used one. Activity patterns as well as their cooccurrences can be identified through the analysis of process models rather than event logs. Related to this problem, this paper proposes a method for discovering and analyzing activity pattern co-occurrences in business process models. Our results are used for developing a BPM tool which fosters the modeling of business processes based on the reuse of activity patterns. Our tool includes an inference engine which considers the patterns co-occurrences to give design time recommendations for pattern usage.
\end{abstract}

\section{INTRODUCTION}

Process-aware information systems (PAIS) prove to be efficient tools for the design and automation of business processes. Business process is a set of (structured) activities which jointly perform a particular business goal. Such activities are related to specific business functions or process fragments (e.g., notification, approval) having a well defined semantics (Thom, 2006a, Thom, 2006b). In particular, a certain process fragment or business function (e.g., enabling document approval) may occur several times within one or different process models. That means multiple logical copies of the same process fragment may be used with same or different parameterization (e.g. approval by a single actor or by multiple actors). In (Thom, 2009) such fragments are represented as workflow activity patterns: Request for Activity Execution without Answer, Request for Activity Execution with Answer, Approval, Notification, Decision-making and Information Request.

An example of activity pattern is the unidirectional performative pattern. This pattern represents a unidirectional message as described in (zur Muehlen, 2002). A sender uses unidirectional performative messages to request the execution of a particular activity from a receiver (e.g., human or software agent) involved in the process. The sender continues execution of his part of the process immediately after having sent the request. The complete set of activity patterns on which this work is based is described in (Thom, 2006b, Thom, 2009).

Generally, multiple activity patterns can be composed to a process model using workflow patterns (e.g., Sequence, AND-Split, AND-Join, XOR-Split). Through an empirical study, in which 214 real-world process models were analyzed, the existence of seven activity patterns has been confirmed (cf. Thom 2008a, Thom, 2008b, Thom, 2009). In that approach it was shown that the analyzed process models can be completely designed based on the aforementioned patterns; i.e., the set of identified activity patterns has been necessary as well as sufficient to design the 214 process models, at least at a certain level of granularity. This pattern set is closer to the vocabulary abstraction level which business processes are usually described by domain experts. 
Though it is known that pattern utilization improves the quality and performance of process modeling, contemporary BPM tools like Intalio, ARIS Toolset and WBI Modeler do not support process designers in defining, querying and reusing activity patterns as building blocks for business process modelling (OASIS, 2006).

In this paper we present preliminary results concerning the discovery and analyses of the cooccurrences of the activity patterns in real-world process models. Our goal is to use the results of this analysis for developing a BPM tool, which fosters the modeling of business processes based on the reuse of activity patterns. The results of our analysis can be further used by this tool to suggest a ranking of the activity patterns best suited to succeed the last applied pattern in a process design, facilitating the modeling phase and leading to more standardized and less error prone process models.

In order to perform the analysis we apply a knowledge discovery on databases (KDD) process. It is a process which involves theories and tools to help humans to extract knowledge from large, growing digital datasets. Its main purpose is to identify valid patterns, potentially useful and understandable from a set of data. In this context, data constitute a set of facts; and patterns are expressions in some language describing a subset of the data or a model applicable to them. It can be split into 5 main phases: 1) data selection; 2) data preprocessing; 3) data transformation; 4) data mining, and 5) result validation (Fayyad, 1996).

In particular, we implement a process model mining tool (BPM mining tool for short) to be used for identifying activity patterns co-occurrences. Our miner allows analysing process models instead of event logs as proposed in literature (Günther, 2008), (Aalst, 2003). This can be considered as a very important functionality to automatically identify activity patterns co-occurrences in real-world process models.

The remainder of this paper is organized as follows: In section 2 we discuss related work. Section 3 presents the data mining technique as well as the algorithm used to perform the mining of the activity patterns co-occurrences in real process models. Section 4 applies the KDD process for mining the process models. In this section we also introduce the BPM mining tool we are developing and some results generated by this tool when being applied to real process models. Finally, Section 5 concludes the paper with a summary outlook.

\section{RELATED WORK}

Recently, a variety of workflow patterns was suggested for capturing different aspects in PAISs including control and data flow, resources, process change, and exception handling (Aalst, 2003), (Weber, 2007). However, there has not yet been a mapping of activity patterns onto process (meta) models and process modeling tools respectively.

Concerning workflow patterns, tool support is provided by YAWL (Aalst, 2005), which uses extended workflow nets as building blocks for workflow specifications. Multiple extended workflow nets involved in a workflow specification can be connected to each other by composite tasks.

The PICTURE approach proposes a set of 37 domain specific process building blocks (Becker, 2007). More precisely, these building blocks are used by end users in Public Administrations to capture the process landscape and are also specific to this domain). Finally, ProCycle presents an approach implementing process change patterns in ADEPT2 (Weber, 2009).

\section{SELECTING A DATA MINING METHOD AND ALGORITHM}

If the goal of a KDD process is to predict the behaviour of some variables, utilization of models, which only describe or group the data, will not be useful. Besides that the kind of learning, the task to be done, the type of repository to be mined, and knowledge representation must be considered during the selection of the data mining technique (Brand, 1998), (Goebel, 1999), (Han, 2001). Table 1 presents these aspects and respective values which were considered in the selection of the data mining algorithms used in our approach.

Table 1: Aspects which were considered in the selection of the data mining method.

\begin{tabular}{|l|l|}
\hline Data mining aspect & Value \\
\hline Model Type & Predictive \\
\hline Learning Type & Unsupervised/Batch \\
\hline Task to be done & Association Analysis \\
\hline Repository to be mined & Process models database \\
\hline Knowledge Representation & White box \\
\hline
\end{tabular}

Based on Table 1 we have studied the algorithms for association rules discovery. This technique is best suited for association analysis and is a predictive one. This choice was made, because what we are looking for is a future prediction based on already occurred facts. In our case, the occurred 
facts are represented by process models partially designed, while the goal is to discover (or to predict) the next activity pattern to be used in the modeling of these process models.

The technique is also based on unsupervised/batch learning, i.e., it allows to prepare a set of processes in a file and send this file to the data mining algorithm in a batch mode.

Besides that, the white box concept guarantees that the results when applying this technique are easily understandable by humans so that we can make some manual inferences with the mined information (Agrawal, 1993).

Given a set of transactions, where each transaction is a set of items, an association rule is an expression $A$ (antecedent) $=>C$ (consequent), where $A$ and $C$ are sets of items. The intuitive meaning of such a rule is that transactions in the database, which contain the items in $A$, tend to contain the items in $C$ as well. (Agrawal, 1993).

The main advantages of association rules that motivate their use in our approach are as follows: (a) they are easily understood by humans; (b) they are used to represent empirical associations; (c) through special measures (support and confidence) it becomes possible to evidence how useful mining results are (Han, 2001).

A workflow process model can be understood as a graph with a predefined semantics. In this context, patterns are identified in recurrent subgraphs within a set of graphs (e.g., a set of processes models). Given a set of graphs, a graph mining algorithm searches for substructures which satisfy some criteria (e.g., minimum frequency of patterns and minimum confidence). Based on recurrent substructures, association rules can be derived. In this context, we have selected the Frequent SubGraph (FSG) algorithm to be used in our approach (Kuramochi, 2004). We choose FSG due to its good performance results, scalability, good documentation and simple and well defined I/O.

\section{APPLYING KDD FOR MINING PROCESS MODELS}

In the data selection step of our KDD process we analyzed 190 process models. Most analyzed models had been created with the Oracle tool or an UML based process modeling tool. Altogether, the considered process models stem from 12 different organizations and are related to different application domains. Note that the mining is performed considering the process models and not their execution logs as done in other approaches (see (Tristão, 2008), (Aalst, 2003)).

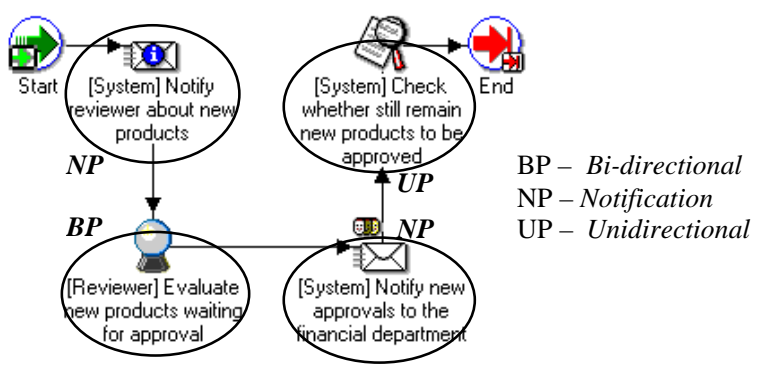

Figure 1: Example of activity patterns identification.

To mine process models, first of all we need to transform the model such that the mining algorithm can extract the needed information. In this preprocessing phase the 190 process models were analyzed and the activity patterns they use were identified. Each activity or partial order of activities was labelled as activity pattern.

\begin{tabular}{|c|c|}
\hline \multicolumn{2}{|c|}{$\begin{array}{l}\text { t \# Example - } 1 \text { - Approval process for a marketing } \\
\text { campaign of a new product }\end{array}$} \\
\hline $\mathrm{V} \odot \mathrm{S}$ & \\
\hline $\mathrm{V} 1 \mathrm{~N}$ & $t-$ transaction \\
\hline $\mathrm{v} 2 \mathrm{~B}$ & $v$ - graph node (activity pattern) \\
\hline $\mathrm{V} 3 \mathrm{~N}$ & $u$ - edge \\
\hline $\mathrm{V} 4 \mathrm{U}$ & $S$ - start node \\
\hline $\mathrm{V} 5 \mathrm{EO}$ & $N$ - notification \\
\hline $\begin{array}{lllllll}1 & 0 & 5 & 0 \\
u & 0 & 1 & N\end{array}$ & $B$ - bi-direcional pattern \\
\hline $42 \mathrm{~N}$ & $U$ - unidirectional pattern \\
\hline $\begin{array}{llll}0 & 1 & 2 & \mathrm{~N} \\
\mathrm{u} & 2 & 3 & \mathrm{~N}\end{array}$ & EO - end node \\
\hline $\mathrm{u} 34 \mathrm{~N}$ & \# - comment sign \\
\hline u $45 \mathrm{~N}$ & \\
\hline
\end{tabular}

Figure 2: Example of process transformation.

In the data transformation phase we mapped each process model together with the identified activity patterns, to the format required by the FSG algorithm (cf. Section 3). For example, the process model shown in Fig. 1 was transformed to the format depicted in Fig. 2. Each node has an incremental index and a label which indicates the activity pattern this node represents. Edges refer to the nodes they connect and have a label. All edges have the $N$ (Normal) label since we are not making any differentiation between them.

In the mining phase the FSG algorithm is executed. As a result of the mining, the frequent subgraphs in the analyzed process models are identified as well as the support value of each sub-graph within the set of process models and their parent-child relation. This information is stored in a file similar to the input file, since the frequent patterns are graphs as the input process models are. 


\subsection{On a Process Model Mining Tool}

In order to analyze the results from the mining phase we developed a BPM mining tool which allows to automatically execute specific parts of the data mining and the evaluation steps of the KDD process. The tool receives a set of normalized activity patterns as input and then simulates their construction with the use of the mined cooccurrences patterns. In this context co-occurrence is a recurrent construction of activity patterns within process models (e.g., the pattern pair DECISION $\rightarrow$ NotifiCATION).

To evaluate the usefulness of the activity patterns co-occurrence, the tool simulates a user modeling the input processes. In this modeling all processes are re-constructed activity by activity. For each activity insertion, the tool generates a recommendation list. This list is composed by rules describing how the process can evolve according to the partial model designed so far and the cooccurrences found on the data mining phase. These rules have on their antecedent and consequent parts of process models. Fig. 3 shows two examples of rules (see item $b$ ).

As the tool receives as input the complete process, it knows all intermediary stages of the modeling phase as well as how process elements must be added to completely design it. The order in which elements are added to the process is obtained from the order they appear in the input file. Therefore, the tool knows what would be the user's choice on the recommendation list if he was modeling the process with the help of it since the order of elements insertion is specified. For instance, the process shown in Fig. 2 could be part of an input for the BPM mining tool. With that description the tool simulates a user which models that process by first adding an edge between the start node and the notification pattern. Then, it adds an edge connecting this notification pattern to a bidirectional performative one. After that, the bidirectional pattern is connected to other notification pattern, which is then connected to a unidirectional performative pattern, which is finally connected to an end node.

The output of the BPM mining tool is a summary containing calculations regarding the simulation of the input models designed. It can be used to analyze how useful the information on the co-occurrence of activity patterns can be to predict and thus to foster the design of process models. Sample outputs are:

- the total number of co-occurrence rules found in the data mining phase;
- the number of steps of user's rule selection simulated, i.e., number of times that a recommendation list was created and the appropriate rule was searched on it;

- a ranking with the total number of steps where the rule on position $n$th of the recommendation list would be chosen by the user, i.e., the total times that the rule on first (and second, ..., $n$ th) position of the recommendation list would be selected by a user when modeling the process;

- the total number of rules of size $i$ that were chosen in the simulation (in this analysis the size of a rule is the number of edges that its antecedent model has).

\subsection{Implementation of a Process Model Mining Tool}

Based on the recurrent activity patterns found in the mining phase, our BPM mining tool creates all rules of interest with their respective support and confidence. In future, we intend to implement some filters within this class, or in a new class called by, in order to filter the created rules according to specific criteria. This filtering contributes to avoid the creation of too many rules and improves the performance of the algorithm. Currently, the used filtering creates only one step rules. Item $b$ of Fig. 3 shows two rules of this type. These rules have a partial process model as antecedent and have on the consequent the same process model with one more edge and eventually a node. It is assumed that each process model may evolve only by adding new edge in each design step. When adding a new edge we have two situations: a) the new edge closes a cycle on the process, so that no new nodes are added; or b) the new edge connects a node of the partial model designed to a new activity pattern (node). In case of $b$ a new node is added to the model as well. By doing so, we assure that all created rules represent an evolution of the model of one step. Note that this approach is easier for a user to understand when comparing to rules which may present greater modifications of the partial model designed so far. If we allow to create rules which introduce greater changes of the process they will hardly match all user intentions. Besides that, this is not our purpose, i.e., we do not want to present an almost complete process to the user. Instead, we want to interactively help on each pattern insertion to the process model, so the rules are kept simple and easily readable.

Our tool also simulates the design of the input process models. It has an inference engine which receives a partially modelled process as input and 
then tries to match the complete process, or part of it, with the antecedent of the rules as created in the data mining phase. All matching rules are returned in the ranking list of activity pattern co-occurrences, so they represent a possible evolution for the design of the partial model. Currently, the rules are ordered by their size and confidence. Thus, rules which match the largest part of the partial model are presented first. If the rules have same size, the one presenting the greatest confidence will be shown first.

Fig. 3 shows a fragment of the process model we introduced in Fig. 1, (see $a$ ). Fig. 3 also presents a recommendation list with two rules to continue the modeling of the process from the model fragment designed so far (see $b$ ). The partial process model is considered to construct this recommendation list. It contains all rules that match entirely or partially the model presented in $a$.

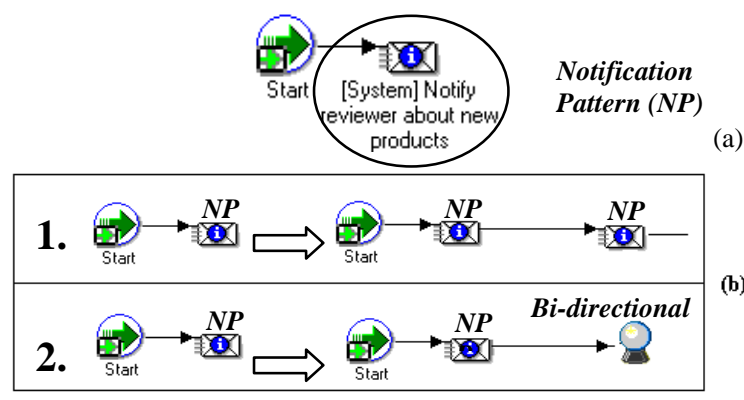

Figure 3: Modeling evolution.

Based on the complete process model (cf. Fig. 1 ), our tool knows that, in this case, if the user is assisted by the recommendation list when designing the process, he will choose rule number two, since it represents an evolution of the partial model by adding a new bi-directional performative pattern connected to the already existing notification pattern.

\subsection{Evaluating the Discovered Patterns}

To evaluate the patterns discovered with the KDD process we have done a randomly stratified partitioning of the available processes. We reserved $1 / 3$ to pass to the Tester class once the cooccurrences patterns are mined over the other $2 / 3$ of the processes. It was considered a minimum support of 3, i.e., if three process models contain the same co-occurrence of activity patterns it will be, then, considered a pattern in our data mining step. Doing so we assure that the co-occurrences found during data mining step are not obtained from processes we use to verify our approach. Thus, we can check whether they are predictive enough for 'never seen' process models.

In our experiment we had observed that the 470 steps of rule selection were simulated, i.e., 470 times a co-occurrence ranking of activity pattern was generated and the appropriate rule which describes the model evolution was searched. This ranking has an average size of 26 rules. We can observe that on almost $20 \%$ of the simulated steps, the rule which correctly predicts the evolution of the model is on the first three positions of the ranking. Accumulating the values until the $10^{\text {th }}$ position we have a correct prediction in more than $35 \%$ of the simulated steps. Considering that we have 6 activity patterns, the probability of correctly predict the next activity pattern, without additional information, is around $16.67 \%$. Further, we need to predict where this new activity pattern will be inserted. For instance, in a model with 2 nodes, this probability decreases to the half. In a model with 10 nodes, this probability is ten times lower.

We can also see that the majority of the rules that correctly predicts the model evolution are rules of size 1,2 or 3 . This conclusion can further be used to filter the generated rules to return rules of only these sizes and reduce the amount of rules on the ranking. We expect that this filter will improve the prediction rate of the inference engine.

After evaluating the mined patterns we have reexecuted the process using all the available process models. At this time, we used the complete set of models to execute the data mining step and then simulated their construction. 1425 simulation steps were executed. On the first three positions of the ranking we had a correct prediction rate of more than $30 \%$ while on the $10^{\text {th }}$ first positions this rate goes to more than $50 \%$. The percentage of unmatched rules has decreased from $41 \%$ on before execution to $20.5 \%$. Considering the average size of 11 nodes in the processes models analysed, we have around $1.52 \%$ of chances to correctly predict the next pattern to be inserted in a model and where to insert it if no prior information is used. With three chances, i.e., creating a list of three possible next patterns, we have $4.55 \%$ of chances to get a match. Increasing from $4.55 \%$ to $30 \%$ is a great gain and this illustrates the power of using mined information to predict the future on our experiments.

\section{SUMMARY}

This paper reports on the use of KDD in the development of a BPM mining tool, which allows 
mining process models based on activity patterns as highly relevant. The functionalities of this tool can be considered very important: a) after having identified the activity patterns in the process models the tool can count the recurrences of each pattern as well as their co-occurrences; b) the inference engine of our BPM mining tool can give design time recommendations for any new processes being modelled, which ease process modelling based on already mined information; c) we can use our tool for conducting a series of experiments in which we compare process modeling with and without activity pattern support as well as investigate different process classes and their most recurrent cooccurrences and; d) finally, the basic concepts behind this tool (e.g., the inference engine) can be added as extensions to existing BPM tools.

As future work, we aim at extending our tool with a module to update the frequency of activity patterns co-occurrences and corresponding raking of recommendations based on the user modeling. This update will be done on-the-fly as new models are developed aided by the inference engine. Thus, we aim at increasing the accuracy of the recommendations for each pattern co-occurrence.

In addition we intend to investigate methods which allow the automatic identification of activity patterns in real process models.

\section{ACKNOWLEDGEMENTS}

The authors would like to acknowledge the Coordination for the Improvement of Graduated students (CAPES), the DBIS of the Ulm University. (Germany), and the Informatics Institute of the UFRGS (Brazil).

\section{REFERENCES}

Agrawal, R., Imielinski, T., Swami, A., (1993). Mining association rules between sets of items in large databases. In: Proc. of the ACM SIGMOD, p.207-216.

Becker, J., Pfeiffer, D., Räckers, M. (2007). Domain Specific Process Modelling in Public Administrations The PICTURE Approach. Proc. EGOV’07, pp. 68-79

Brand, E., Gerritsen, R., (1998). Data Mining and Knowledge Discovery, Oct. 1998.

Fayyad, U., Shapiro-Piatetsky, G., Smyth, P., (1996). From Data Mining to Knowledge Discovery in Databases, AI Magazine, v.17, n.3, p. 37-54, [s.l.].

Goebel, M., Gruenwald L., (1999). A survey of data mining and knowledge discovery software tools. In:
SIGKDD Explorations, v. 1, p. 20-33.

Günther, C.W., Rinderle-Ma, S., Reichert, M., van der Aalst, W.M.P., Recker, J., (2008). Using Process Mining to Learn from Process Changes in Evolutionary Systems. In: Int. Journal of Business Process Integration and Management, 3(1):61-78.

Han, J., Kamber, M., (2001). Data Mining: Concepts and Techniques, Morgan Kaufmann, 550 p., San Diego.

Kuramochi, M., Karypis, G., (2004). An Efficient Algorithm for Discovering Frequent Subgraphs, In: IEEE Transaction on Knowledge and Data Eng., Minneapolis, v.16, n.9, p. 1038-1051.

Li, C. Reichert, M., Wombacher, A., (2008). Discovering Reference Process Models by Mining Process Variants. proc. In: Proc. of ICWS, Beijing. pp. 45-53.

OASIS. (2006). Web Services Business Process Execution Language. Version 2.0.

Thom, L. H., Iochpe, C., Amaral, V., Viero, D., (2006a). Towards Workflow Block Activity Patterns for Reuse in Workflow Design. In: WfMC Workflow Handbook. pp. 249-260.

Thom, L.H., (2006a). A Patterns -based Approach for Business Process Modeling. PhD Thesis. UFRGS: Porto Alegre, Brasil.

Thom, L.H., Reichert, M., Chiao, C., Iochpe, C., Hess, G., (2008b). Inventing Less, Reusing More and Adding Intelligence to Business Process Modeling. In: Proc. of DEXA, Turin, LNCS 5181, pp. 837-850.

Thom, L. H., Iochpe, C., Reichert, M., Weber, B., Droop, M., Nascimento, G., Chiao, C. M., (2008c). On the Support of Activity Patterns in ProWAP: Case Studies, Formal Semantics, Tool Support. In: iSys. Vol 1, No 1, pp. 27-53.

Thom, L. H., Reichert, M., Iochpe, C., (2009). Activity Patterns in Process-aware Information systems: Basic Concepts and Empirical Evidence. In: IJPIM.

Tristão, C., Ruiz, D. D., Becker, K., (2008). FlowSpy: exploring Activity-Execution Patterns from Business Processes. In: Proc. of Simp. Brasileiro de Sistemas de Informação, Rio de Janeiro, 2008. v. 1. p. 152-163.

van der Aalst, W.M.P., ter Hofstede, A.H.M., Kiepuszewski, B., Barros, A., (2003). Workflow Patterns. In: Distributed. and Parallel. Database, 14(3): 5-51.

van der Aalst, W.M.P., (2005). YAWL: Yet Another Workflow Language. Information Syst., 30(4):245-275.

Weber, B., Rinderle, S., Reichert, M., (2007). Change Patterns and Change Support Features in ProcessAware Information Systems. In: Proc. of CAiSE'07, LNCS 4495, pp. 574-588.

Weber, B., Reichert, M., Wild, W., Rinderle-Ma, S., (2009). Providing Integrated Life Cycle Support in Process-Aware Information. In: Int'l Journal of Cooperative Information Systems, 18 (1).

zur Muehlen, M. (2002). Workflow-based process controlling: foundations, design, and application of workflow-driven process information systems. Logos. 